\title{
Population pharmacokinetics of tobramycin
}

\author{
L. AARONS ${ }^{1}$, S. VOZEH ${ }^{2}$, M. WENK ${ }^{2}$, Ph. WEISS ${ }^{2}$ \& F. FOLLATH ${ }^{2}$ \\ ${ }^{1}$ Pharmacy Department, University of Manchester, Manchester and ${ }^{2}$ Division of Clinical Pharmacology, \\ Department of Medicine, University Hospital, Kantonsspital, Basel, Switzerland
}

1 Population pharmacokinetic parameters of tobramycin were determined in a heterogenous group of 97 patients using serum samples drawn for the routine monitoring of tobramycin concentrations, following multiple dosing regimens.

2 To describe the accumulation kinetics of tobramycin a two-compartment pharmacokinetic model was required. The best fit to the data was obtained when drug clearance $\left(1 \mathrm{~h}^{-1}\right)$ was related linearly to creatinine clearance (proportionality constant: $0.059 \pm$ $\left.0.002 \times \mathrm{CL}_{\mathrm{cr}}\left(\mathrm{ml} \mathrm{min}^{-1}\right)\right)$ and initial volume of distribution (l) was related linearly to body weight (proportionality constant: $0.327 \pm 0.014 \times$ body weight $(\mathrm{kg})$ ). The intersubject variability in these two parameters was $32 \%$ and $3 \%$, respectively, whilst the residual or intrasubject variability amounted to $21 \%$ of the tobramycin concentration. The terminal half-life of tobramycin, $26.6 \pm 9.4 \mathrm{~h}$, was appreciably shorter than previously reported.

3 The population pharmacokinetic model was validated against data obtained from 34 independent patients and the predicted and observed concentrations were found to be in good agreement. The population pharmacokinetic model was used to design a priori dosing recommendations for tobramycin.

Keywords tobramycin population pharmacokinetics therapeutic drug monitoring

\section{Introduction}

Aminoglycosides represent an important class of antimicrobial agents used primarily in patients with severe infections due to gram-negative bacteria. They are usually administered as intermittent bolus doses (or short infusions) and it appears that the achievement of a sufficiently high serum concentration within each dosage interval plays an important role with respect to their efficacy (Powell et al., 1983; Moore et al., 1984a,b; Gerber et al., 1986). On the other hand the available data indicate that a continuous exposure to relatively high serum concentrations (reflected by high trough or average drug levels in each dosing interval) over a prolonged period of time increases the risk of the two severe sideeffects, nephrotoxicity and ototoxicity (Mawer et al., 1974; Smith et al., 1986). Thus, for the effective and safe use of aminoglycoside antibiotics it is important to achieve sufficiently high maximum serum concentrations and at the same time avoid prolonged continuous exposure to high drug serum levels.

Pharmacokinetic principles can be applied to achieve this goal. Population pharmacokinetic parameters, i.e. parameters that describe the average dose-concentration relationship and its between-patient variability in the patient population actually treated with the drug, represent, for two reasons, important information with respect to the effective use of pharmacokinetic principles for dosage optimization of tobramycin. Because of its narrow therapeutic index and the availability of a rapid assay, tobramycin is frequently monitored, particularly in patients with 
Table 1 Patient demographic data

\begin{tabular}{llll}
\hline & & Main study & Prospective study \\
\hline Number of patients & 97 & 34 \\
Weight & mean & & \\
$(\mathrm{kg})$ & median & 66.5 & 66.8 \\
& s.d. & 66.8 & 66.5 \\
& range & 12.5 & 10.9 \\
Age & mean & $42-120$ & $45-87$ \\
(years) & median & 50.6 & \\
& s.d. & 51.0 & 57.8 \\
& range & 19.0 & 62.0 \\
Sex & & $16-85$ & 20.6 \\
& male/female & $52 / 45$ & $17-85$ \\
Creatinine & mean & & \\
clearance & median & 67.9 & $22 / 12$ \\
(ml min $^{-1}$ ) & s.d. & 65.0 & 73.3 \\
& range & 33.0 & 63.3 \\
& & $10-166$ & 34.4 \\
\hline
\end{tabular}

reduced renal function. Dosing guidelines are based on the pharmacokinetic parameters of the individual patient. However, frequently only one or two serum drug concentrations are available and consequently it is not possible to estimate an individual's pharmacokinetic parameters. In this situation Bayesian techniques, in which population pharmacokinetic parameters are sequentially updated by available serum measurements, have proven successful (Burton et al., 1985). Apart from their use in Bayesian feedback, knowledge of population pharmacokinetics is useful in describing the average dose-concentration relationship and its intra- and interindividual variability. This information can be used to design an optimum $a$ priori dosing regimen that should result in 'therapeutic' concentrations in the majority of patients and to evaluate the need for therapeutic drug monitoring.

It was the purpose of the present study to analyse routinely obtained serum tobramycin concentrations in a large number of patients with a view to estimating the population pharmacokinetic parameters of tobramycin and to determine an a priori dosing regimen in patients with various degrees of renal function impairment.

\section{Methods}

\section{Patients}

Data from 97 patients ( 45 female, 52 male) were included in the analysis (see Table 1). Blood samples were taken in most cases for the routine monitoring of tobramycin concentrations. The dosing history and time of blood sampling were carefully recorded. The duration of therapy in individual patients ranged from 14 to $520 \mathrm{~h}$. Three hundred and twenty-two tobramycin concentrations were available for the data analysis with between 1 and 9 per individual. The concentration data are shown in Figure 1. As can be seen there was a large number of patients with dosing over 10 days, allowing estimates of the accumulation characteristics. Drug concentrations were measured usually 2 and $6 \mathrm{~h}$ after the dose in patients with normal renal function, with additional measurements ( 12 and $24 \mathrm{~h}$ after the dose) in patients with impaired renal function. In eight patients the serum drug concentration was monitored up to 3 days after. cessation of therapy (3-5 samples). The patients had an average age of $51 \pm 19$ years (range 16-85) and an average weight of $66 \pm 12 \mathrm{~kg}$ (range 42-120). Creatinine clearance (estimated from serum creatinine) ranged from 10 to $160 \mathrm{ml}$ $\min ^{-1}$ (average $67 \pm 33$ ). The formula used for the estimation of creatinine clearance $\left(\mathrm{CL}_{\mathrm{Cr}}\right)$ was that proposed by Cockcroft \& Gault (1976):

$$
\mathrm{CL}_{\mathrm{Cr}}=\frac{(150 \text {-age }) \cdot \text { Body weight }}{C_{\mathrm{Cr}}}
$$

where $C_{\mathrm{Cr}}$ is the serum concentration of creatinine in $\mu \mathrm{mol} \mathrm{l}^{-1}$. Only patients with stable renal function were included in the study. For males $10 \%$ is added to and for females $10 \%$ is subtracted from the calculated value of $\mathrm{CL}_{\mathrm{Cr}}$. 


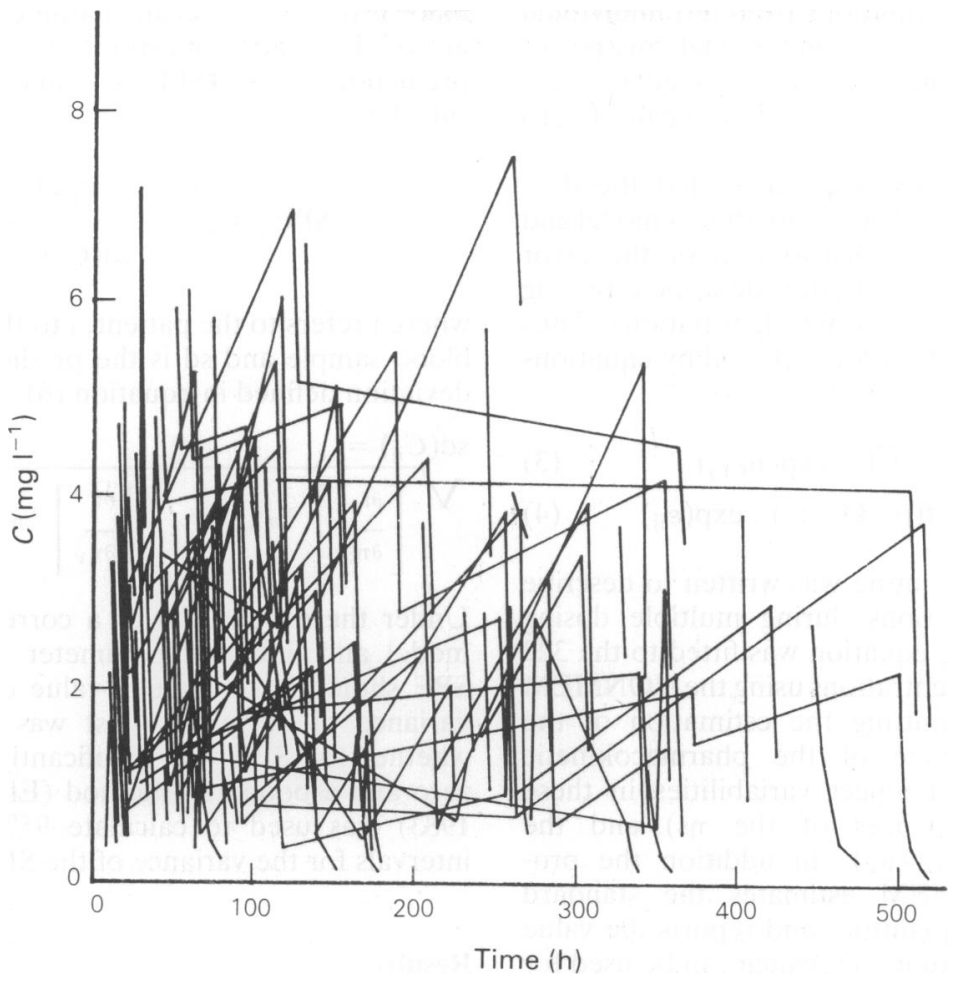

Figure 1 Tobramycin concentration-time data from 97 patients used in the analysis.

Assay

For routine tobramycin monitoring, the serum drug concentrations were measured using an homogeneous enzyme immunoassay $\left(\mathrm{EMIT}^{\circledR}\right.$, Syva Corp., Palo Alto, CA). The within-assay and between-assay coefficients of variation for this method were $7.4 \%$ (at a concentration of $4 \mathrm{mg} \mathrm{l}^{-1}$ ) and $3.6 \%$ (at a concentration of $6 \mathrm{mg} \mathrm{l}^{-} 1$ ), respectively (Francke et al., 1981). In eight patients tobramycin serum concentrations were followed for up to $72 \mathrm{~h}$ after cessation of therapy. These serum samples were measured with a modified radioimmunoassay (Coat-ACount ${ }^{\circledR}$, Diagnostic Products Corp., Los Angeles, CA). In the modified method a 1:51 predilution of the serum samples rather than the 1:401 dilution as described in the manufacturer's instructions was used. The coefficient of variation of the RIA was $7.3 \%$ (at a concentration of $\left.1 \mathrm{mg} \mathrm{l}^{-1}\right)$.

\section{Data analysis}

The analysis was performed with the NONMEM computer programme developed by Beal \&
Sheiner (1979). The individual pharmacokinetic parameters are assumed to arise from a distribution (either normal or log normal) characterized by a population mean and interindividual variance. For example the clearance $(C L)$ of the jth subject can be described by the following relationship

$$
\mathrm{CL}_{\mathrm{j}}=\overline{\mathrm{CL}}+\eta_{\mathrm{CL}}
$$

where $\overline{\mathrm{CL}}$ is the population mean and $\eta_{\mathrm{CLj}}$ is the difference between the population mean and the jth subject. $\eta_{C L}$ is assumed to be a Gaussian random variable with mean zero and variance $\sigma_{\eta}^{2}$. The concentration-time profile in the jth individual can be described by equation (2)

$$
C_{\mathrm{ij}}(\mathrm{t})=\mathrm{f}\left(\mathrm{p}_{\mathrm{j}}, \mathrm{D}_{\mathrm{ij}}, \mathrm{t}_{\mathrm{ij}}\right)+\varepsilon_{\mathrm{ij}}
$$

where $p_{j}$ are the pharmacokinetic parameters (such as clearance, volume etc) of the jth individual, $\mathrm{t}_{\mathrm{ij}}$ is the time of the ith measurement, $D_{\mathrm{j}}$ is the dosing history of that individual and $f$ is the pharmacokinetic model, for example a sum of exponentials. $\varepsilon_{\mathrm{ij}}$ represents the residual departure of the model from the observations 
and contains contributions from intraindividual variability, assay error and model misspecification. As for the $\eta s, \varepsilon$ is assumed to be a random Gaussian variable with a mean of zero and a variance $\sigma_{\varepsilon}^{2}$.

Preliminary analysis revealed that the data were best fitted by a two-compartment model and that the probability distribution of the error terms $\eta$ and $\varepsilon$ was better described by $\log$ normal rather than normal distributions. Thus equations (1) and (2) are replaced by equations (3) and (4).

$$
\begin{gathered}
\mathrm{CL}_{\mathrm{j}}=\overline{\mathrm{CL}} \cdot \exp \left(\eta_{\mathrm{CLj}}\right) \\
C_{\mathrm{ij}}(\mathrm{t})=\mathrm{f}\left(\mathrm{p}_{\mathrm{j}}, \mathrm{D}_{\mathrm{ij}}, \mathrm{t}_{\mathrm{ij}}\right) \cdot \exp \left(\varepsilon_{\mathrm{ij}}\right)
\end{gathered}
$$

A recursive subroutine was written to describe serum concentrations during multiple dosing and the resulting equation was fitted to the 322 tobramycin concentrations using the NONMEM programme permitting the estimation of the population average of the pharmacokinetic parameters, intersubject variabilities in these parameters (variances of the $\eta \mathrm{s}$ ) and the residual variance $\left(\sigma_{\varepsilon}^{2}\right)$. In addition the programme NONMEM estimates the standard errors of these quantities and reports the value of the objective function, which can be used for hypothesis testing. Model comparison was made by testing the difference in $\log$ likelihood values against a chi-squared statistic $(P<0.01$; Beal \& Sheiner, 1979).

The four pharmacokinetic parameters used in the analysis were the clearance, initial volume of distribution $\left(V_{1}\right)$ and the two transfer rate constants, $k_{12}$ and $k_{21}$. In addition the dependence of these parameters on a number of covariates, such as creatinine clearance and body weight was tested.

\section{Validation of the parameter estimates}

To validate the pharmacokinetic model and the estimates of the population pharmacokinetic parameters another set of data which was not included in the original statistical analysis was evaluated. Data from 34 patients were used. The duration of therapy ranged from 1 to 21 days, with greater than 6 days therapy in 10 patients. Body weight ranged from 45 to $87 \mathrm{~kg}$ and the estimated creatinine clearance from 18 to $166 \mathrm{ml} \mathrm{min}^{-1}$. The demographic data for this group of patients are compared to the original group in Table 1 . Serum drug concentrations 2 and $6 \mathrm{~h}$ after the final dose were available for each patient. Based on the patient's creatinine clearance, body weight and dosing history a prediction of the tobramycin concentration was made using the model and parameters described above. For each concentration a standardized prediction error (SPE - equation (5)) was calculated

$$
\mathrm{SPE}_{\mathrm{ij}}=\frac{C_{\mathrm{ij}}-\mathrm{f}\left(\mathrm{p}_{\mathrm{j}}, D_{\mathrm{ij}}, \mathrm{t}_{\mathrm{ij}}\right)}{\operatorname{sd}\left(C_{\mathrm{ij}}\right)}
$$

where $j$ refers to the patient, $i$ to the time of the blood sample and sd is the predicted standard deviation defined in equation (6)

$\operatorname{sd}\left(C_{\mathrm{ij}}\right)=$

$\sqrt{\left[\frac{\partial \mathrm{f}}{\partial \eta_{\mathrm{CL}}}\right]{ }^{2} \cdot \sigma^{2}{ }_{\eta \mathrm{CL}}+\left[\frac{\partial \mathrm{f}}{\partial \eta_{V}}\right]{ }^{2} \cdot \sigma^{2}{ }_{\eta V}+\mathrm{f}^{2} \cdot \sigma_{\varepsilon}^{2}}$

Under the assumption of a correct regression model and unbiased parameter estimates the SPE should have a mean value of zero and a variance of unity. A $t$-test was used to test whether the SPE was significantly different to zero and a bootstrap method (Efron \& Gong, 1983) was used to calculate $95 \%$ confidence intervals for the variance of the SPE.

\section{Results}

As stated in the methods section a twocompartment model was clearly superior to a one-compartment model $(P<0.001)$ and correcting clearance for creatinine clearance $\left(\mathrm{CL}_{\mathrm{Cr}}\right)$ viz.

$$
\mathrm{CL}=\Theta_{1} \cdot \mathrm{CL}_{\mathrm{Cr}}
$$

where $\theta_{1}$ is the parameter that is estimated, also resulted in a highly significant difference in the objective function $(P<0.001)$, and improvement of the goodness of fit as judged by the residual plots, interindividual variability in $\mathrm{CL}$ and the residual variability. The influence of body weight on $V_{1}$ was also significant $(P<0.001)$. Making $V_{1}$ dependent on either body weight raised to a power or lean body mass did not give any improvement over simple proportionality to body weight. There was a tendency for the proportionality constant between $V_{1}$ and body weight to depend on age in that it was larger for patients below the age of 21 years. However, since there were only five patients belonging to the young group the inclusion of age led to poorly determined parameter estimates. Therefore, it was eventually dropped from the analysis. Sex and age did not contribute significantly to any of the other pharmacokinetic parameters. The interindividual variability in 
Table 2 Population pharmacokinetic parameters of tobramycin ${ }^{\text {a.b }}$

\begin{tabular}{lccccc}
\hline \multirow{2}{*}{ Parameter } & \multicolumn{2}{c}{ Population mean } & & \multicolumn{2}{c}{ Interindividual variability } \\
\cline { 2 - 3 } \cline { 5 - 6 } & Estimate & s.e. & & Estimate & s.e. \\
\hline $\mathrm{CL}^{\mathrm{c}}$ & 0.059 & 0.002 & & $32 \%$ & $44 \%$ \\
$k_{12}\left(\mathrm{~h}^{-1}\right)$ & 0.012 & 0.003 & & - & - \\
$k_{21}\left(\mathrm{~h}^{-1}\right)$ & 0.027 & 0.021 & & - & - \\
$V_{1}\left(\mathrm{lg}^{-1}\right)$ & 0.327 & 0.014 & & $3 \%$ & $112 \%$ \\
$\sigma_{\varepsilon}$ & $21 \%$ & $38 \%$ & & & \\
\hline
\end{tabular}

a Estimates of variability expressed as coefficient of variation

b s.e. of variance components $\left(\operatorname{var}(C L), \operatorname{var}(V), \sigma_{\varepsilon}^{2}\right)$ taken as

$\checkmark$ s.e.(estimate)/estimate expressed as a percentage

c Proportionality constant relating creatinine clearance $\left(\mathrm{ml} \mathrm{min}^{-1}\right)$

to drug clearance $\left(\mathrm{h} \mathrm{h}^{-1}\right)$

${ }^{d}$ Residual intraindividual coefficient of variability of the serum concentration

Table 3 Mean and standard deviation of the standardized prediction error in 34 patients receiving tobramycin ${ }^{\mathrm{a}}$

\begin{tabular}{lccccc}
\hline & \multicolumn{2}{c}{$2 h$} & & \multicolumn{2}{c}{$6 h$} \\
\cline { 2 - 3 } \cline { 5 - 6 } & $\begin{array}{c}\text { Mean } \\
(95 \% \text { c.i. })^{b}\end{array}$ & $\begin{array}{c}\text { s.d. } \\
(95 \% \text { c.i. })\end{array}$ & & $\begin{array}{c}\text { Mean } \\
(95 \% \text { c.i. })\end{array}$ & $\begin{array}{c}\text { s.d. } \\
(95 \% \text { c.i. })\end{array}$ \\
\hline $\begin{array}{l}\text { Group I } \\
(n=24)\end{array}$ & 0.16 & 0.88 & & 0.20 & 1.29 \\
$\begin{array}{l}\text { Group II } \\
(n=10)\end{array}$ & $(-0.22,0.54)$ & $(0.64,1.08)$ & & $(-0.36,0.76)$ & $(0.76,1.80)$ \\
$\begin{array}{l}\text { Group I + II } \\
(n=34)\end{array}$ & 0.53 & 0.91 & & 0.26 & 1.46 \\
\hline
\end{tabular}

${ }^{a}$ Group I consists of those patients whose duration of therapy was less than 6 days; Group II duration of therapy greater than 6 days. $n$ is the number of patients in each group.

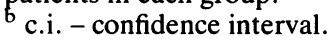

CL (after correction for creatinine clearance) was $32 \%$ (expressed as the coefficient of variation of the population average). The interindividual variability in $V_{1}$ was very small (with a large standard error) after correction for body weight. Including interindividual variability in $k_{12}$ and $k_{21}$ did not improve the fit and therefore these variances were removed from the final analysis. A summary of the results is shown in Table 2.

The two exponents had half-lives of 2.55 (s.e. - 0.12) and $26.6 \mathrm{~h}$ (s.e. -9.4) (for an individual with a creatinine clearance of $100 \mathrm{ml}$ $\min ^{-1}$ ) and the volume of distribution at steady state (for a $70 \mathrm{~kg}$ individual) was $32.9 \mathrm{~L}$ (s.e. 1.0).

In the evaluation of the parameter estimates in the other group of 34 patients $2 \mathrm{~h}$ samples were analyzed separately from $6 \mathrm{~h}$ samples and patients were divided into two groups-those with duration of therapy less than 6 days and those with durations greater than 6 days-to see whether the goodness of prediction was influenced by the time of blood sampling or duration of therapy. The results of this analysis are shown in Table 3. In all cases the SPE was unbiased (i.e. not significantly different from zero) and the variance of the SPE was not significantly different from unity.

\section{Discussion}

In most clinical settings the pharmacokinetics of tobramycin are described by a one-compartment model. Although such a model adequately predicts the serum concentration-time profile of the drug in most patients with normal renal function (disregarding a very short distribution phase) it does not account for accumulation of the drug in 
tissues (which is believed to contribute to its toxicity) and fails to describe adequately the serum concentration-time profile in some patients on multiple dosing, in particular in the presence of renal function impairment (Vozeh et al., 1979; Evans et al., 1980). Schentag et al. (1978) estimated the pharmacokinetic parameters of tobramycin associated with accumulation by fitting a two-compartment model to washout data obtained from patients after terminating a multiple dosage regimen. The parameters obtained by these authors were quite different from the present results in that the estimate of the terminal half-life was very much longer. The main difference between our study and the work of Schentag et al. (1978) is that in the present study the terminal half-life was determined predominantly by the accumulation in tobramycin concentrations after multiple dosing and not from washout data. In fact, the present report is, to our knowledge, the only study in which the accumulation half-life of an aminoglycoside antibiotic was determined directly from drug accumulation during multiple dosing. There are two main possibilities why the estimates of the terminal half-life determined under the two different experimental designs may show large differences. The degree of accumulation of tobramycin based on serum concentrations is, except in patients with severe renal failure, relatively small and consequently accurate estimation of the terminal half-life from these data may be difficult. On the other hand, in washout studies the serum drug concentrations are very low after the first $24 \mathrm{~h}$ $\left(<0.05 \mathrm{mg} \mathrm{l}^{-1}\right)$ and it is conceivable that renal handling of the aminoglycoside antibiotics may be different at these extremely low serum concentrations compared with the concentrations achieved during a course of treatment. A saturable tubular reabsorption that is effective only at very low serum concentrations could, for example, result in a long lasting persistence of the serum concentrations in washout studies with a substantially shorter accumulation half-life determined under multiple dosing. There are indications in the literature that for some aminoglycosides nonlinear elimination processes are, in fact, present in the washout studies. A large discrepancy is observed between the estimates of the terminal half-life determined in the same subject from urinary data and the serum drug concentration-time course. Laskin et al. (1983) found in healthy subjects, in whom the elimination of gentamicin and netilmicin was followed over 1 week after multiple dosing, terminal half-lives of 94 and $155 \mathrm{~h}$, respectively, based on the serum concentration measurements compared with values of 58 and $46 \mathrm{~h}$ determined from the urinary excretion data. These authors suggest that nonlinear elimination processes become limiting at concentrations below $0.05 \mathrm{mg} \mathrm{l}^{-1}$. Similar discrepancies can be noted in studies in patients performed by Kahlmeter $e t$ al. with gentamicin (1978a) and tobramycin (1978b). These findings contradict the notion that the extremely long half-life found in the washout studies is only due to slow release of the drug from tissues. Obviously, further work is needed to clarify these problems.

Several authors have tried to correlate volume of distribution to some function of body weight, such as lean body mass. Studies in morbidly obese patients show that there is some distribution of tobramycin into adipose tissues (Schwartz et al., 1978; Bauer et al., 1983) and consequently it is not surprising that in our studies lean body mass or other functions of body weight offered no definitive advantage over straight body weight to explain volume of distribution. We should like to point out, however, that in our study population (which was an unselected population of patients treated with tobramycin) only few patients were morbidly obese. It has been reported that younger patients require a higher loading dose on a $\mathrm{mg} \mathrm{kg}^{-1}$ basis than older patients (King et al., 1985), a trend seen in our studies. The reason for this age effect is not clear but it may reflect the different distribution of tissue masses as a function of age. The estimate of the intersubject variability in the volume of distribution was smaller than expected. However, the earliest blood sample was not taken until $2 \mathrm{~h}$ after the dose to avoid serum drug concentration measurements during the early distribution phase. We suspect that this might be the reason that there was little information in the data concerning the variability in $V_{1}$. Most of the intersubject variability is accounted for by $\mathrm{CL}$. The problem of distinguishing, in our data, between the variability in clearance and volume of distribution might have been aggravated by the fact that NONMEM uses a regression model which is linearized with respect to the $\eta \mathrm{s}$ to determine the interindividual variances (Beal \& Sheiner, 1979). Therefore we have little faith in the actual estimate of the intersubject variability in the volume of distribution and believe that it is meaningful only in the context of the whole population pharmacokinetic model.

Because of the theoretical problems discussed above and the fact that population pharmacokinetic analysis is usually performed in an exploratory way it is important that the final model and the parameter estimates obtained in such 
Table 4 Dosing recommendations for tobramycin

\begin{tabular}{cccccc}
\hline & \multicolumn{2}{c}{ First 48 $h$} & & \multicolumn{2}{c}{ Maintenance } \\
\cline { 2 - 3 } \cline { 5 - 6 } $\begin{array}{c}\text { Creatinine clearance } \\
\left(\text { ml min }^{-1}\right)\end{array}$ & $\begin{array}{c}\text { Dose } \\
\text { Do of } \\
\text { loading } \\
\text { dose })\end{array}$ & $\begin{array}{c}\text { Dosing } \\
\text { interval }(h)\end{array}$ & & $\begin{array}{c}\text { Dose } \\
(m g)\end{array}$ & $\begin{array}{c}\text { Dosing } \\
\text { interval }(h)\end{array}$ \\
\hline 100 & 100 & & & & \\
75 & 80 & 8 & & 120 & 8 \\
50 & 93 & 12 & & 90 & 8 \\
30 & 60 & 12 & & 54 & 12 \\
20 & 80 & 24 & & 70 & 24 \\
15 & 67 & 24 & & 54 & 24 \\
10 & 60 & 24 & & 35 & 24 \\
\hline
\end{tabular}

a A loading dose of $2.1 \mathrm{mg} \mathrm{kg}^{-1}$ is used in each case with a conservative upper limit of $150 \mathrm{mg}$

studies are validated on a separate data set collected in another group of patients. This validation, using data in 34 patients has revealed that the prediction of both the average concentration and its variability are unbiased. Therefore it can be concluded that the model and population pharmacokinetic parameters determined in this study can be used satisfactorily to predict average concentrations and variability in patients receiving tobramycin therapy.

\section{Derivation of a priori dosing regimens}

The estimates of the population pharmacokinetic parameters allowed us to design $a$ priori dosage recommendations for tobramycin. This was done by exploring the average concentration profile and its variability (intra- and interindividual) under different dosing schemes with the help of computer simulations. The objective was to maintain sufficiently high maximum concentrations in the beginning of treatment before the dose can be individually adjusted on the basis of drug concentration measurements. Using conventional pharmacokinetic principles this is readily achieved. However the population analysis allowed us to determine not only the average response but also the fraction of the population that would likely meet our objectives. According to literature data a peak level above $5-6 \mathrm{mg} \mathrm{l}^{-1}$ is associated with higher clinical efficacy (Moore et al., 1984a,b). It was therefore decided to aim to achieve peak drug concentrations above $6 \mathrm{mg} \mathrm{l}^{-1}$ in the majority of patients but maintain average concentrations, within a dosing interval (as a measure of accumulation potential), below $4 \mathrm{mg}^{-1}$. The dosing recommendations are given in Table 4. Note that different dosage is recommended for initial treatment and after $48 \mathrm{~h}$. Using a loading dose of $2.1 \mathrm{mg} \mathrm{kg}^{-1}$ (with a conservative upper limit of $150 \mathrm{mg}$ in order not to overdose severely overweight patients) it is predicted that $72 \%$ of patients will achieve an initial concentration greater than $6 \mathrm{mg} \mathrm{l}^{-1}$. After loading, the dosing regimen depends on renal function. Two examples are illustrated in Figures 2 and $3(70 \mathrm{~kg}$ body weight is assumed in both cases). In the case of the subject with creatinine clearance $100 \mathrm{ml} \mathrm{min}^{-1}$ (Figure 2) it is recommended that $150 \mathrm{mg}$ be given every $8 \mathrm{~h}$ for $48 \mathrm{~h}$ by which time $82 \%$ of patients will have maximum concentrations in excess of $6 \mathrm{mg} \mathrm{l}^{-1}$. In the other case (Figure 3 ) with a patient with a creatinine clearance of $10 \mathrm{ml} \mathrm{min}^{-1}$ a loading dose of $150 \mathrm{mg}$ is again given but the dosing interval is increased to $24 \mathrm{~h}$ and, to obtain a similar pattern to that shown in Figure 2, $90 \mathrm{mg}$ is given at $24 \mathrm{~h}$. Thus, based on the Monte Carlo simulations, a somewhat higher dose is needed than generally recommended (Sarubbi \& Hull, 1978) to achieve sufficiently high peak drug concentrations in most patients. We believe that this recommendation is relatively safe in view of the fact that the risk of toxicity depends on duration of treatment (Mawer etal., 1974; Smith et al., 1986) and is therefore expected to be low during the first days of therapy. After the first $48 \mathrm{~h}$, however, the dose should be reduced using serum drug concentration measurements to individually adjust dosage. The dosing rate is reduced (see Table 3 last column) to $120 \mathrm{mg}$ every $8 \mathrm{~h}$ in the case of normal renal function, and to $35 \mathrm{mg}$ every $24 \mathrm{~h}$ in the patient with a $\mathrm{CL}_{\mathrm{Cr}}$ of $10 \mathrm{ml}$ $\min ^{-1}$, resulting in $95 \%$ of patients having average concentrations below $4 \mathrm{mg}^{-1}$. The reason for dose reduction after $48 \mathrm{~h}$ is the increased risk of excessive drug accumulation and 


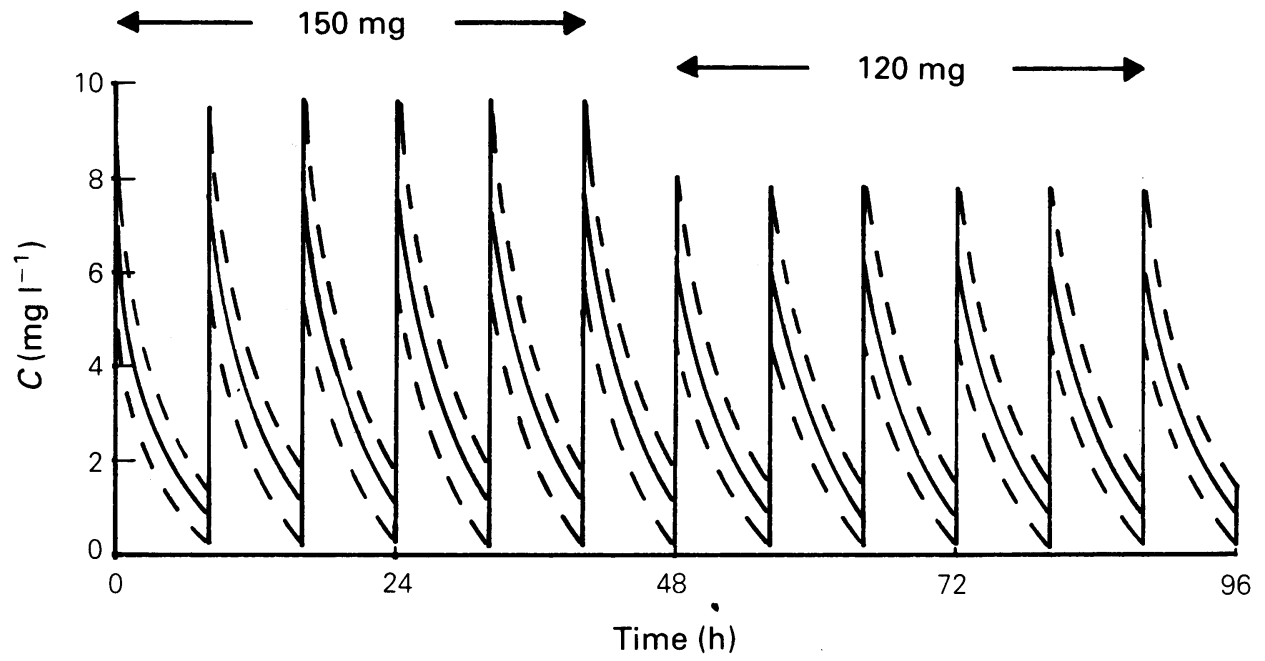

Figure 2 Predicted ( \pm 1 s.d. i.e. approximate $68 \%$ prediction interval) tobramycin concentration-time profile for patients with creatinine clearances of $100 \mathrm{ml} \mathrm{min}^{-1}$.

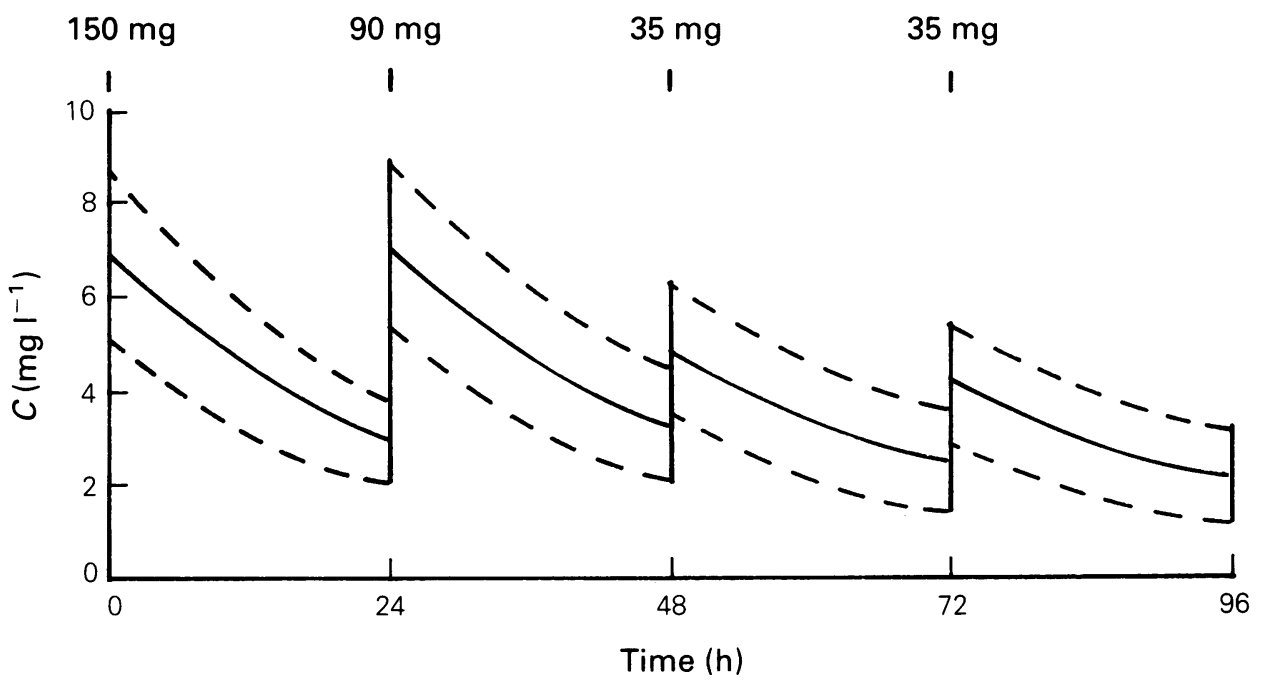

Figure 3 Predicted ( \pm 1 s.d. i.e. approximate $68 \%$ prediction interval) tobramycin concentration-time profile for patients with creatinine clearances of $10 \mathrm{ml} \mathrm{min}^{-1}$.

toxicity with prolonged treatment producing high average and trough concentrations. As can be seen in Figures 2 and 3, although relatively safe $(<5 \%$ of patients are expected to have average concentrations above $4 \mathrm{mg} \mathrm{l}^{-1}$ ), the dosing regimen will fail to achieve the desired maximum concentration of $6 \mathrm{mg}^{-1}$ in a large proportion of patients (43\% in Figure 2 and $98 \%$ in Figure 3 ). These results clearly show that individual dose adjustment based on serum drug concentration measurements is needed for safe and effective therapy and that the dosing of tobramycin is difficult in patients with severely impaired renal function. On the other hand since toxicity and drug accumulation takes time, a relatively high dose can be administered initially which will guarantee effective concentrations in most patients. The period of $48 \mathrm{~h}$ during which the high dose is administered was chosen on the basis of our experience that within this time individual measurements of the serum drug concentration will be usually available, allowing 
appropriate dose adjustment. Although trough concentrations are commonly used to avoid excessive accumulation of aminoglycoside antibiotics we believe that the average concentration (area under the serum concentration-time curve divided by the length of the dosing interval) is more meaningful in this situation (Barza et al., 1978; Follath et al., 1981; Wenk et al., 1984). The main advantage of the average concentration compared with trough concentrations is that it reflects the accumulation potential independent of the length of the dosing interval. This is an important consideration in view of the fact that to achieve effective maximum concentrations in patients with impaired renal function (without excessive overdosing) different lengths of dosing interval have to be used depending on the patient's renal function (Table 4). The choice of the upper limit of $4 \mathrm{mg} \mathrm{l}^{-1}$ is somewhat arbitrary,

\section{References}

Barza, M. \& Lauermann, M. (1978). Why monitor serum levels of gentamicin? Clin. Pharmacokin., 3, 202-215.

Bauer, L. A., Drew Edwards, W. A., Patchen Dellinger, E. \& Silmonowitz, D. A. (1983). Influence of weight on aminoglycoside pharmacokinetics in normal weight and morbidly obese patients. Eur. J. clin. Pharmac., 24, 643-647.

Beal, S. L. \& Sheiner, L. B. (1979). NONMEM users guide. San Francisco: University of California.

Burton, M. E., Brater, C., Chen, P. S., Day, R. B., Huber, P. J. \& Vasko, M. R. (1985). A Bayesian feedback method of aminoglycoside dosing. Clin. Pharmac. Ther., 37, 349-357.

Cockcroft, D. W. \& Gault, H. (1976). Prediction of creatinine clearance from serum creatinine. Nephron, 16, 31-41.

Efron, B. \& Gong, G. (1983). A leisurely look at the bootstrap, the jackknife and cross-validation. The Am. Statist., 37, 36-48.

Evans, W. E., Taylor, R. H., Feldman, S., Crom, W. R., Rivera, G. \& Yee, G. C. (1980). A model for dosing gentamicin in children and adolescents that adjusts for tissue accumulation with continuous dosing. Clin. Pharmacokin., 5, 295-306.

Follath, F., Wenk, M. \& Vozeh, S. (1981). Plasma concentration monitoring of aminoglycosides. J. Antimicrob. Chemother., 8 (Suppl A): 37-43.

Francke, E. L., Srinivasan, S., Labthavikul, P.\& Neu, H. C. (1981). Rapid, reproducible enzyme immunoassay for tobramycin. J. clin. Microbiol., 13, 93-96.

Gerber, A. U., Brugger, H. P., Feller, C. Stritzko, T. \& Stalder, B. (1986). Antibiotic therapy of infections due to Pseudomonas aeruginosa in normal and granulocytopenic mice: comparison of murine and human pharmacokinetics. J. infect. Dis., 153, 90-97. because very little quantitative information is available in the literature about the relationship between tobramycin toxicity and drug accumulation in the body. In a patient with normal renal function the average concentration at steady-state under the recommended dosage (Table 4, Figure 2) is expected to be $2.6 \mathrm{mg} \mathrm{l}^{-1}$. The upper limit of $4 \mathrm{mg} \mathrm{l}^{-1}$ represents, therefore, a value that is associated with a $50 \%$ increase in drug accumulation compared with an average patient receiving an effective dosing regimen. It is also close to the average concentration in a patient with a trough concentration of $2 \mathrm{mg} \mathrm{l}^{-1}$ (and a peak of $6 \mathrm{mg} \mathrm{l}^{-1}$ ) receiving tobramycin every $8 \mathrm{~h}\left(3.7 \mathrm{mg} \mathrm{l}^{-1}\right)$.

This work was supported in part by the Professor Max Cloëtta Foundation, the Swiss National Research Foundation and the Royal Society.
Kahlmeter, G., Jonsson, S. \& Kamme, C. (1978a). Longstanding post-therapeutic gentamicin serum and urine concentrations in patients with unimpaired renal function. A pharmacokinetic evaluation. J. Antimicrob. Chemother., 4, 143-152.

Kahlmeter, G., Jonsson, S. \& Kamme, C. (1978b). Multiple-compartment pharmacokinetics of tobramycin. J. Antimicrob. Chemother., 4 (Suppl A): 5-9.

King, C. H., Greger, R. J. \& Ellner, J. J. (1985). Pharmacokinetics of tobramycin and gentamicin in abusers of intravenous drugs. Antimicrob. Agents Chemother., 27, 285-290.

Laskin, O. L., Longstreth, J. A., Smith, C. R. \& Lietman, P. S. (1983). Netilmicin and gentamicin multidose kinetics in normal subjects. Clin. Pharmac. Ther., 34, 644-650.

Mawer, G. E., Ahmad, R., Dobbs, S. M., McGough, J. G., Lucas, S. B. \& Tooth, J. A. (1974). Prescribing aids for gentamicin. Br. J. clin. Pharmac., 1, 45-50.

Moore, R. D., Smith, C. R. \& Lietman, P. S. (1984a). The association of aminoglycoside plasma levels with mortality in patients with gram-negative bacteremia. J. infect. Dis., 149, 443-448.

Moore, R. D., Smith, C. R. \& Lietman, P. S. (1984b). Association of aminoglycoside plasma levels to therapeutic outcome in gram-negative pneumonia. Am. J. Med., 77, 657-662.

Powell, S. H., Thompson, W. L., Luthe, M. A., Stern, R. C., Grossniklaus, D. A., Bioxham, D. D., Groden, D. L., Jacobs, M. R., DiScenna, A. O., Cash, H. A. \& Klinger, J. D. (1983). Once-daily vs continuous aminoglycoside dosing: efficacy and toxicity in animal and clinical studies of gentamicin, netilmicin and tobramycin. J. infect. Dis., 147, 918-932.

Sarubbi, F. A. \& Hull, J. H. (1978). Amikacin serum 
concentrations: prediction of levels and dosage guidelines. Ann. intern. Med., 89 (Part 1): 612618.

Schentag, J. J., Lasezkay, G. Cumbo, T. J., Plaut, M. E. \& Jusko, W. J. (1978). Accumulation pharmacokinetics of tobramycin. Antimicrob. Agents Chemother., 13, 649-656.

Schwartz, S. N., Pazin, G. J., Lyon, J. A., Ho, M. \& Pasculle, A. W. (1978). A controlled investigation of the pharmacokinetics of gentamicin and tobramycin in obese subjects. J. infect. Dis., 138, 499-505.

Smith, C. R., Moore, R. D. \& Lerner, S. A. (1986).
Aminoglycoside ototoxicity - interaction between duration of therapy and plasma trough levels. Clin. Pharmac. Ther., 39, 229.

Vozeh, S., Spring, P., Wenk, W. \& Follath, F. (1979). Changes in apparent half-life of gentamicin and tobramycin without detectable changes in creatinine clearance. Br. J. clin. Pharmac., 7, 629-631.

Wenk, M., Vozeh, S. \& Follath, F. (1984). Serum level monitoring of antibacterial drugs. Clin. Pharmacokin., 9, 475-492.

(Received 10 January 1989, accepted 5 June 1989) 